\title{
Jugular, hepatic, and praecordial pulsations in constrictive pericarditis
}

\author{
A. El-Sherif and G. El-Said \\ From the Cardiac Department, Cairo University Hospitals, U.A.R.
}

A study of the pulsatile features in constrictive pericarditis has been attempted in II cases. The diagnosis was confirmed in 5 cases at operation. The expected increase in the frequency of the condition in certain localities is put forward. Venous neck pulsations with particular reference to the pathogenesis of the $x$ and $y$ descents are discussed. Hepatic pulsations previously not sufficiently stressed in this disease are described and are graphically documented. They are found to conform, though to a lesser magnitude, with the venous neck pulsations. Apex cardiography has been utilized to study praecordial pulsations. A reproducible curve was obtained from the praecordium in all cases. The curve appears characteristic of the condition and consists of a small isometric contraction phase, low $E$ point, a wide systolic trough, a small isometric relaxation phase, a steep rapid filling wave (RFW), and a rebound wave, which is followed by a diastolic plateau without or including an ill-defined $A$ wave. The curve is characteristically biphasic and the rebound wave rather than the $E$ point forms its summit. Restoration of the abnormal cardiogram to a normal pattern follows successful pericardiectomy. The genesis of the abnormal curve is discussed.

The incidence of constrictive pericarditis of tuberculous aetiology is expected to be high in countries where this infection is still prevalent. Data from our department indicate that before the era of chemotherapy many patients with tuberculous pericarditis used to die in the active stage before the development of constriction. An early diagnosis is essential for successful operation and the addition of new diagnostic techniques to those already used may be of help. Of the various criteria, jugular venous pulsations have received the greatest attention (Gimlette, 1959; Wood, 1961; Sorour et al., 1963). Some studies on apical pulsations have also been made, utilizing electrokymography (McKusick, 1952), accelerator ballistocardiography (Mounsey, 1957, 1959), and impulse cardiography (Boicourt, Nagle, and Mounsey, 1965). Hepatic pulsations, on the other hand, have not - received much attention.

The present work aims at a detailed study

2 of the hepatic and praecordial movements in conjunction with the jugular pulsations in constrictive pericarditis. The simplicity of apex cardiography and its reliability in the diagnosis of several cardiac disorders have stimulated us to use it in the diagnosis of this Received 22 May 1970. condition. Apex cardiography refers to the low-frequency displacement curve in the range of $0 \cdot 10-20 \cdot 0$ cycles per second recorded at the point of maximum impulse in midexpiration, in the left lateral position (Benchimol and Dimond, 1963). The technique has recently been extended to the study of other pulsations and has been termed the praecordial cardiogram (El-Sherif, Saad, and ElSaid, 1969).

\section{Subjects and methods}

Nine men and two women were studied. Except in one case there was no residual pericardial effusion or active inflammation. The diagnosis was made clinically, radiologically, and electrocardiographically according to established criteria (White, 1951; Gimlette, 1959; Wood, 1961; Sorour et al., 1963). The diagnosis was confirmed haemodynamically through right-sided catheterization in 6 cases and at operation in the remaining 5. No case with annular constriction, such as that described by Mounsey (1959), was encountered. Pericardial calcification and atrial fibrillation were present each in three cases. Clinical examination was made by each author separately, then by both together for the final decision and before the graphic recordings of the jugular, hepatic, and praecordial movements were undertaken.

Jugular phlebograms and external hepatograms were recorded while the patients were in the sit- 
ting, semirecumbent, or recumbent position, whichever brought maximum pulsations. The graphs were analysed and compared with the pressure curves obtained during cardiac catheterization from the superior vena cava, inferior vena cava, and hepatic veins.

Praecordial movements were recorded during held expiration over areas where pulsations could be detected as described by Benchimol and Dimond (1963) and Ginn et al. (1967). Lead II of the electrocardiogram and a phonocardiogram were simultaneously recorded for timing. Before recording each tracing it was ascertained on an oscilloscope to fulfil the following criteria: (a) an upward deflection approximately synchronous with the $R$ wave of a simultaneously recorded electrocardiogram; (b) a sharp nadir (O point) occurring after the $T$ wave of the electrocardiogram and followed by the diastolic filling waves.

Several successive tracings were obtained on a multichannel Elema Mingograph $42 \mathrm{~B}$, at a paper speed of 25 and $50 \mathrm{~mm}$./sec. Good postoperative records were obtained in only 3 cases. Persistent tenderness of the chest wall after operation and the development of postoperative scar tissue made recording difficult in the rest of the cases.

The records were studied in the light of the criteria given by Benchimol, Dimond, and Carson (196I), Benchimol and Dimond (1963), and Tafur, Cohen, and Levine (1964) for the normal curve. A normal curve (Fig. I) shows: (I) A systolic wave that forms an out-thrust throughout systole, which begins with a rapid rise corresponding to the isometric contraction phase, reaches a sharp tent-like peak ( $E$ point or beginning of ventricular ejection), and descends at first steeply (maximum ventricular ejection) then gradually to a plateau (reduced ventricular ejection). (2) A diastolic wave that begins after the second sound with a steep downward deflection (isometric relaxation) to the $\mathrm{O}$ point (opening of the $\mathrm{AV}$ valve) after which it rises first rapidly then slowly (rapid and slow filling waves) till the presystolic or A wave of the next cycle. The latter wave is caused by displacement of the ventricular wall by atrial contraction.

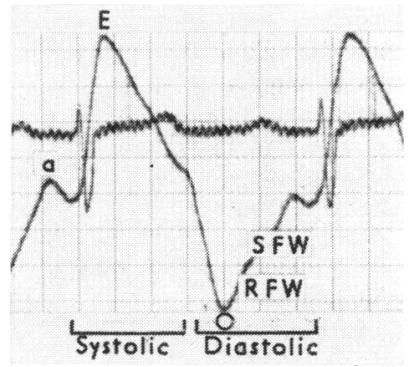

FIG. I Normal apex cardiogram with simultaneously recorded electrocardiogram. a, presystolic wave; $E$, ejection; $O$, o point; $R F W$, rapid filling wave; SFW, slow filling wave.

\section{Results}

Jugular pulsations Jugular venous pulsations were observed in all the cases in one position or the other. In 4 cases with marked venous congestion the pulsations were seen only in the sitting position in 3 and in the standing position in the fourth. A diastolic dip immediately after the radial pulse was seen in all cases, and an additional descent of a variable degree was seen during systole in most of them.

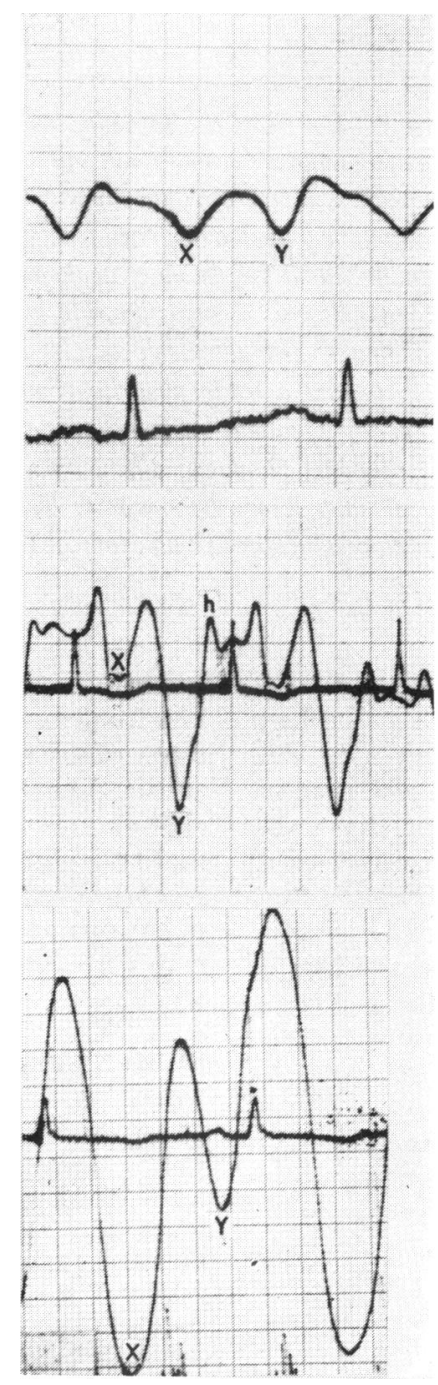

FIG. 2 fugular phlebograms with simultaneously recorded electrocardiogram from three cases showing both the $x$ and $y$ descents. In the upper tracing the $x$ is equal to the $y$; in the middle with atrial fibrillation the $x$ is smaller and in the lower tracing with sinus rhythm it is deeper. In the middle tracing the rebound or ' $h$ ' wave is shown. 
External phlebograms were obtained from all the cases and uniformly showed both $\mathrm{x}$ and $y$ descents (Fig. 2). In 3 cases the $y$ was deeper than the $x$ descent, in 5 both were equal, and in the remaining 3 the $x$ descent was more prominent. Cases in which the $y$ descent was deeper were the 3 cases with atrial fibrillation in the series. No relation was found between the depth of the different waves and the age of the patient, duration of the illness, degree of venous congestion, right atrial pressure, or presence of pericardial calcification. Comparison of the external phlebograms with the superior vena caval and right atrial tracings of the 6 cases catheterized showed exactly similar configurations.

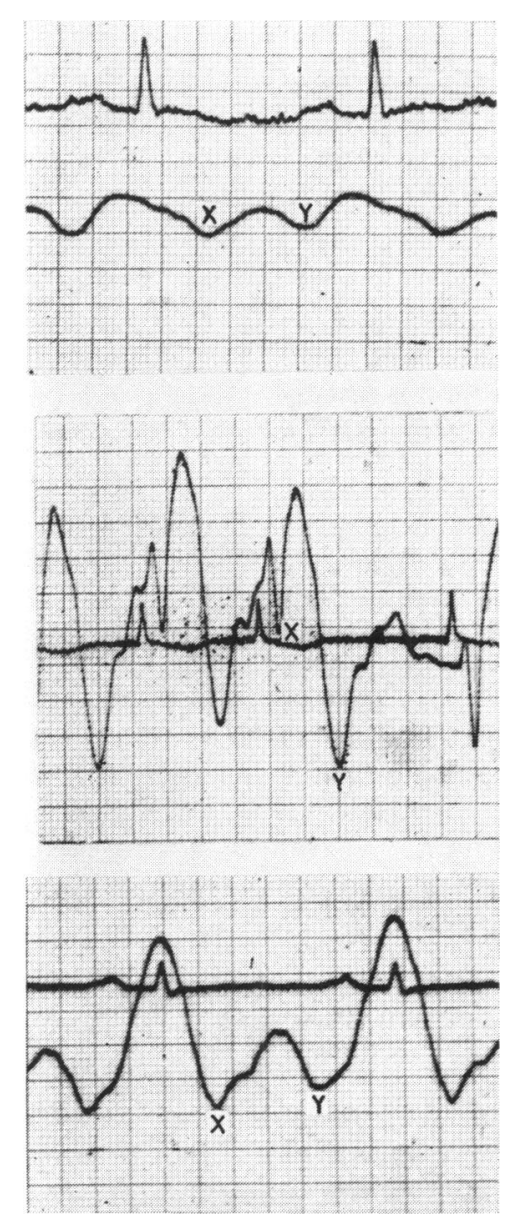

FIG. 3 Hepatograms with simultaneously recorded electrocardiogram from three patients showing the same phenomena as those in Fig. 2. The $x$ and $y$ descents are equal in the upper tracing; the $x$ is deeper in the lower and shallower in the middle tracing.
Hepatic pulsations Liver pulsations were felt in 6 patients, prominent in 4 and faint in 2. They were felt better in the recumbent than in the semirecumbent position. Two cases with free pulsations had pericardial calcification. In 5 patients the pulsation was felt as a diastolic dip after the radial pulse, and coincided with the third heart sound. In the sixth patient it was felt as a systolic dip simultaneously with the radial pulse.

Hepatic tracings (Fig. 3) recorded from the 6 cases showed $\mathrm{x}$ and $\mathrm{y}$ descents. Both waves were equal in 3 cases. The $y$ descent was deeper in 2, while the $x$ descent was more prominent in one. The latter case was the one with hepatic pulsation felt as systolic collapse. The configuration of the hepatic tracings was similar to the phlebograms and to the inferior vena caval and hepatic venous tracings of the corresponding cases, except that the external hepatograms were of smaller magnitude and with less evident $C$ waves. The latter were better identified in the external phlebograms and internal tracings.

Praecordial pulsations Inspection and palpation showed systolic retraction over and inside the region of the supposed apex followed by a diastolic out-thrust in ro out of the II cases. No forward movement was present during systole in the neighbourhood of the systolic retraction or parasternally. In the remaining case no pulsations were visible or palpable.

Praecordial cardiograms were obtained for 9 cases only, and no pulsations could be recorded in the remaining 2. Pulsations were recorded from the cases with pericardial calcification and from the one in the active stage. Mapping of the whole praecordium was attempted and any praecordial movement was recorded. Measurement studies of Benchimol and Dimond (1963) were difficult, but the following alterations characterized the records in all the cases (Fig. 4 and 5).

(I) The individual components of the cardiogram were less demarcated than those of the normal heart.

(2) The total amplitude of the record was small. This was confirmed by the increase which occurred after operation.

(3) The systolic configuration recorded from all the cases consisted of a small isometric contraction component that rose to a low $\mathrm{E}$ point, which was followed by a steep descent ending in a wide trough throughout systole. Near the end of this trough and coinciding with the second heart sound a small positive wave was usually identifiable.

(4) The diastolic configuration found in all 
the cases consisted of a short continuation of the trough during the isometric relaxation from the second sound to an ill-defined $O$ point. The curve thereafter showed the rapid filling wave (RFW) as a steep rise to a high summit, from which it continued commonly after a slight drop as a series of shallow oscillations or plateau with no well-defined slow filling or A waves. The combination of the systolic trough and the high diastolic plateau gives the praecordial curve a characteristic 'biphasic appearance'. Such a curve was uniformly recorded over any praecordial pulsations present. The rise or overshoot of the rapid filling wave above the level of the plateau corresponds by measurement to the ' $h$ ' wave of the corresponding right atrial and ventricular tracing. The latter is analogous to

FIG. 4 Apex cardiogram of two cases of constrictive pericarditis with simultaneously recorded electro- and phonocardiograms. DP, onset of diastolic plateau. Coinciding with the $R$ wave of the electrocardiogram and with the first sound is the small isometric contraction component and the low $E$ point, followed by the steep descent and the wide trough. With the second sound, $S_{2}$, a small positive wave can be identified on the trough. The steep $R F W$ is especially noticeable in the upper tracing and the overshoot or ' $h$ ' wave is noticeable especially in the lower tracing. The shallow oscillations following the overshoot are shown in the lower tracing, but no SFW or a waves can be observed.
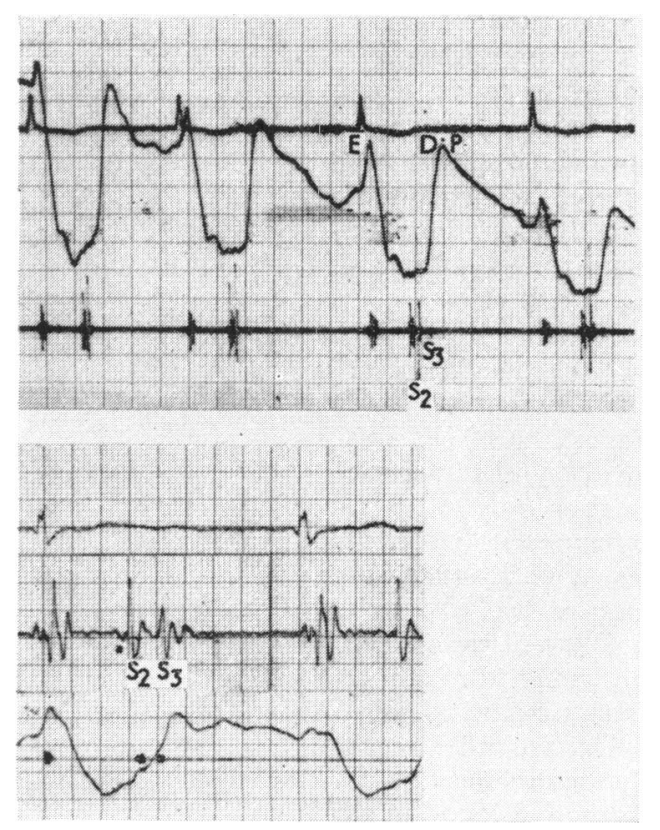
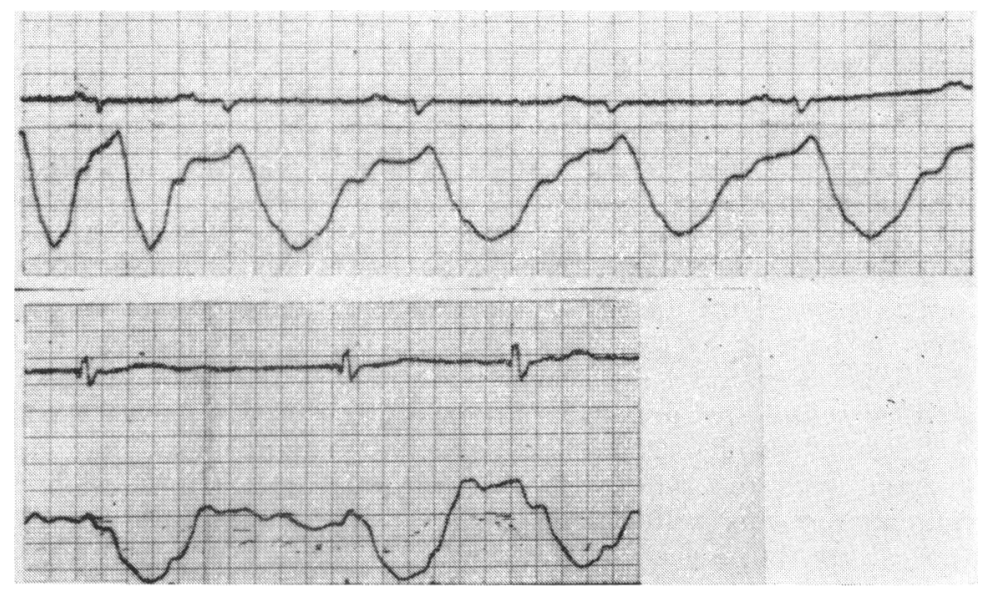

FIG. 5 Apex cardiogram from another two cases, showing the low $E$ point, the systolic trough, and the diastolic plateau. The overshoot ' $h$ ' wave is shown in the lower tracing to constitute its summit. The combination of the systolic trough and the high diastolic plateau gives the curve its biphasic appearance (lower tracing).

the one described in the jugular pulse by Hirschfelder (1907) in subjects with loud third sound. The third heart sound is usually registered along the rapid filling wave (RFW).

Postoperative cardiograms Restoration of the curve after pericardiectomy towards the normal pattern has been striking. The total amplitude increased and the systolic and diastolic components regained their normal pattern with an A wave, isometric contraction phase, high $\mathrm{E}$ point, rapid and slow ejection phases, isometric relaxation phase, sharp $O$ point, and rapid and slow filling waves (Fig. 6).

\section{Discussion}

Jugular pulsations are invariably present in constrictive pericarditis (Gimlette, 1959; Wood, I96I; Sorour et al., 1963). Hepatic pulsations received little attention and Friedberg (1966) stated that the liver did not pulsate in systole.

In our cases jugular pulsations were present in all the patients. In 4 cases, however, venous congestion was so severe that the pulsations were only demonstrable in the sitting or standing positions.

According to Sawyer et al. (1952) there is no obstruction at the mouths of the venae cavae in constrictive pericarditis. This allows the characteristic changes in the right atrium to be faithfully transmitted to the superior 


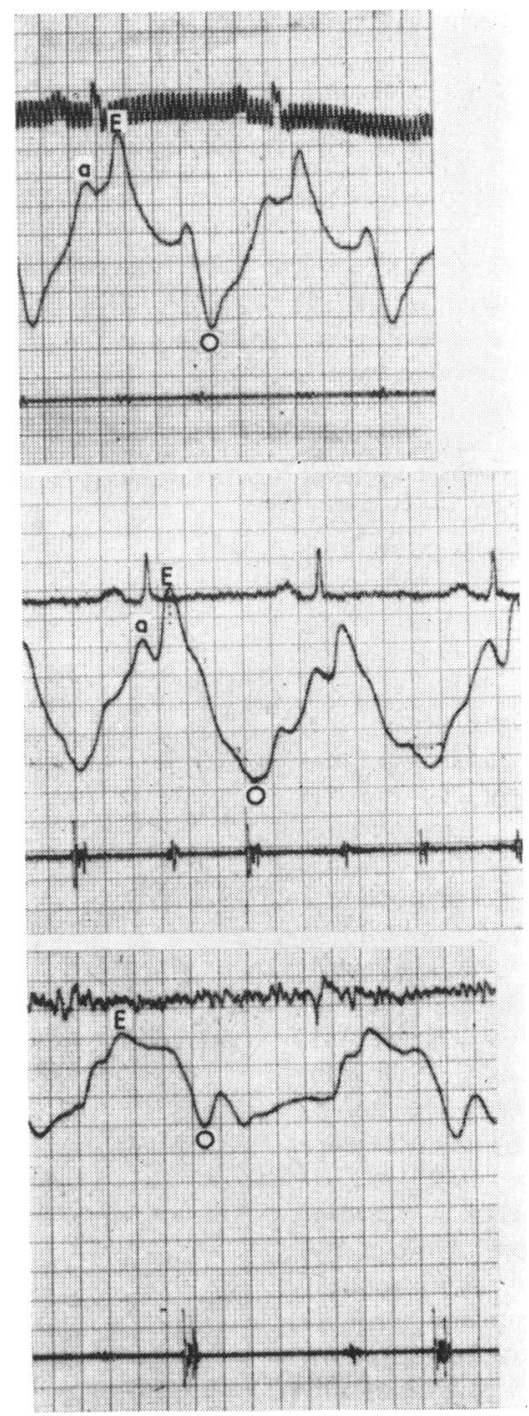

FIG. 6 Postoperative cardiograms from three cases in the series. The upper and lower tracings are of the two cases in Fig. 5. They show increase in amplitude; the well-defined presystolic $a$ wave, the $E$ and $O$ points, and the diastolic wave are apparent. No overshoot or ' $h$ ' wave is present and the $E$ point represents the summit of the tracing.

and inferior venae cavae (Fig. 2). These changes were detailed by Hansen, Eskildsen, - and Götzsche (195r), Wilson et al. (1954), Wood (196r), and Sorour et al. (1963).

In all the cases an early diastolic dip following the radial pulse was seen and coincided with the third heart sound. This sign was described long ago by Friedreich in 1864 but gained popularity after its emphasis by Wood in I96r. This diastolic dip coincides with the $y$ descent in the external phlebograms, superior vena caval, and right atrial tracings. Its steepness is attributed to the rapid drop of the high venous pressure through a nonobstructed tricuspid valve. Because of the fixed capacity of the ventricle, maximal ventricular filling occurs rapidly during the first part of diastole and the $y$ descent is followed by an acute rise which may create a rebound or ' $h$ ' wave that travels up the right atrium and superior vena cava. The latter wave was seen in the right atrial and superior vena caval tracings but was not recorded in all the external jugular phlebograms. It is the high rapid ascent after the $y$ and the rebound or ' $h$ ' wave that imparts depth to the diastolic trough.

Among our cases another descent in the jugular pulse was visualized during systole in most of the cases but was recorded in all. This wave coincided with the corresponding $x$ descent in the superior caval and right atrial tracings. The presence of $x$ descent in right atrial tracings in constrictive pericarditis has been described repeatedly and forms with the y descent a characteristic $M$ - or W-shaped appearance (Hansen et al., I95I; Wilson et al., 1954; Wood, I96I; Sorour et al., 1963). The transmitted $\mathrm{M}$ - or W-shaped pulsation in the neck observed in our cases has been reported also by Gimlette (1959).

The relative amplitude of $\mathrm{x}$ and $\mathrm{y}$ descents in our patients was variable. In the 8 cases with sinus rhythm the $x$ descent was equal to the $y$ in 5 cases and deeper in 3. In the 3 cases with atrial fibrillation the $x$ was less prominent than the $y$. Various explanations were given for the prominent $\mathbf{x}$ descent. Gibson (1959) considered it an atypical feature and ascribed it to the rapid ejection of blood from within the rigid pericardium resulting in lowering of the subsequent $\mathrm{V}$ wave and the AV gradient. Absence of the third heart sound in his cases was taken as evidence to support his explanation. Wood (I96I) attributed the $x$ descent to exceptional descent of the base during systole when the lateral walls of the ventricles have difficulty in moving inwards. We favour Wood's explanation and consider the presence of a prominent $x$ descent equal to or greater than the $y$ a common feature if sinus rhythm is present. Though Gibson (1959) reported that the deep $x$ descent occurs irrespective of the cardiac rhythm we only encountered it in cases with sinus rhythm. That the $\mathrm{x}$ descent becomes less prominent in the presence of atrial fibrillation can be explained by loss of atrial 
contraction which is required for the normal closure of the atrioventricular valves (Little, I95I) or by the absence of atrial relaxation (Nixon and Polis, 1962).

Wood in 196r has described the deep $x$ descent in cases with active disease but it occurred in quiescent cases among our series.

Conspicuous congestion of the neck veins may occur in both constrictive pericarditis and tricuspid regurgitation. The presence or absence of an $\mathrm{x}$ descent in such cases would be a helpful sign in differentiating one condition from the other especially if sinus rhythm is present. The $x$ descent is characteristically absent in cases of severe tricuspid regurgitation.

Hepatic pulsations detectable clinically in a case of constrictive pericarditis were reported by us in a previous publication (Sorour et al., 1963). In the present series pulsations were detected in 6 cases (Fig. 3). They were felt as a diastolic collapse in 5 cases and as a systolic collapse in the sixth. They were better felt in the recumbent than in the semirecumbent position, when liver congestion became less. The discussion on the neck venous pulsations applies equally to the hepatic pulsations. Tracings comparable to those of the right atrium and superior vena cava were obtained from the hepatic veins. The inability to detect hepatic pulsations in all cases and the damped external hepatograms without clear $C$ waves can be explained by the greater amount of hepatic congestion especially in the semirecumbent position.

From the above discussion it appears that the configuration of the neck and hepatic venous pulsations rather than the degree of congestion is more important in diagnosis.

In nearly all our cases inspection of the praecordium showed replacement of the normal cardiac impulse by an evident systolic retraction followed by a diastolic bulge over and medial to the region of the supposed apex. White (1951) and Gimlette (1959) did not refer to any cardiac pulsations in constrictive pericarditis, while Evans (1956) described the apical impulse as either 'not visible' or 'improperly defined'. Wood et al. (I95I) described 'a diastolic heart beat' in constrictive pericarditis characterized by an apical diastolic thrust and a loud protodiastolic sound.

'Apical' pulsations in constrictive pericarditis were repeatedly studied by different methods by McKusick (I952), Mounsey (1957, 1959), and Boicourt et al. (1965), but not by apex cardiography before the present study. The earlier studies entailed only few cases from which no definite conclusions could be made. Our series is larger, and praecordial cardiograms were obtained from most of the cases, including those with pericardial calcification.

A curve with a constant characteristic pattern was regularly obtained from over the pulsatile area in the praecordium (Fig. 4 and 5). Medial to this area pulsations were either absent or weak but of the same pattern. The systolic component of the cardiogram showed deviations from normal in the form of a small isometric contraction phase, a low $\mathrm{E}$ point descending rapidly to a wide trough which was maintained throughout systole.

The diastolic component also showed characteristic changes. The isometric relaxation phase did not appear as the normal downward deflection but as almost a horizontal line. It terminated at the $O$ point, after which the curve ascended abruptly with an overshoot which corresponds to the ' $h$ ' wave of the right ventricular pulse. This wave is most probably the cause of the early praecordial tap, 'knock', or 'shock' described by Schnabel (1966) and Friedberg (1966). The diastolic overshoot, and not the E point, characteristically represents the summit of the tracing. After the overshoot the tracing continued as a plateau at the time interval of the slow filling wave. The $A$ wave is absent as a separate wave and is submerged in the diastolic plateau. Boicourt et al. (1965), using impulse cardiography, reported a diastolic bulge following the systolic retraction over and medial to the apex, but no individual components were described.

In three of our cases praecordial cardiograms were repeated after pericardiectomy. The increase in amplitude and the normalization of the curve with the appearance of its various components were striking (Fig. 6). This fact adds weight to the diagnostic significance of the preoperative cardiograms.

Boicourt et al. (1965) attributed the systolic retraction to the probable existence of adhesions between the pericardium and the anterior chest wall. They ascribed the diastolic impulse in their cases associated with annular constriction to the abnormally large outward movement of the free portion of the anterior right ventricular wall as a result of tethering of the heart over the AV groove and right ventricular outflow tract. Our findings make such explanation unlikely. In 5 of our cases with definite diastolic bulge seen in praecordial tracings the constriction was seen at operation to be of the generalized and not of the annular type.

The praecordial cardiogram in constrictive pericarditis can be readily explained in the light of the origin of its various components and the haemodynamic disturbances that 
occur in the disease. According to Luisada and Magri (1952), the low frequency tracings of the apex cardiogram are the result of several factors, of which the main ones are: (I) Movement of the heart and especially the apex towards the chest wall during cardiac contraction. (2) Volume change of the heart with decrease of the ventricular mass during ejection and its increase during diastole.

Tafur et al. (1964) consider the apex cardiogram to record motions of the chest wall produced by movements of the heart and intrinsic volume pressure changes in its chambers. Coulshed and Epstein (1963) consider the initial upstroke of the apex cardiogram (isometric contraction phase) to be probably produced by increase in the tension of the cardiac muscle leading to alteration of its shape and forward rotation of the apex. When left ventricular volume decreases with ejection of blood the plateau part of the curve is formed. In constrictive pericarditis the dense pericardial sac and the adhesions between its visceral and parietal layers diminish or prevent cardiac movements in systole and diastole (White, 195I). This has been demonstrated by fluoroscopy and roentgenkymography (Stewart, Carty, and Seal, 1943). The praecordial cardiogram in such cases will record mainly the part due to volume changes. This explains the small isometric contraction phase, the low $\mathrm{E}$ point, and the small isometric relaxation phase, which are mainly the result of movement. The volume changes in systole, on the other hand, together with the absence of forward rotation of the apex, explain the systolic retraction and trough.

The diastolic events also reflect exactly the - volume changes produced by the pathophysiological alterations present in the disease. Encasement of the ventricles allows maximal filling in the first part of diastole. The filling abruptly ends when the fixed capacity of the nondistensible ventricle is rapidly reached,

- and this explains the abrupt ascent of the RFW. The rapid halting of blood creates the ' rebound or ' $h$ ' wave in the atrial, ventricular, and sometimes the pulmonary artery pressure curve (Sorour et al., 1963) and the overshoot of the RFW in the praecordial cardiogram. Comparison of the intracardiac and the exter-

nal cardiographic tracings demonstrated that the ' $h$ ' wave in the intracardiac tracings coin-

- cided with the overshoot of the external cardiograms. After the overshoot no further significant filling occurs and a diastolic plateau is recorded. The absence of the a wave, which represents further ventricular filling as a result of atrial contraction, can be readily explained by the inability of the maximally filled ven- tricle to accept any extra blood in late diastole. Gillick (1959) described characteristic electrokymographic changes in constrictive pericarditis due to the abrupt halting of the diastolic filling process by the mechanical restriction of the lateral motion of the ventricle and interference with the waves of both the isometric contraction and relaxation phases.

That these changes are merely the result of the mechanical hindrance offered by the fibrous and calcified pericardium is evident from the restoration of the cardiogram to normal pattern (Fig. 6) after successful pericardiectomy.

The diagnosis of constrictive pericarditis is not always easy, and difficulties may arise with such conditions as myocarditis and myocardiopathies. It remains to be settled whether this praecordial cardiogram will be of help in differentiating these disorders. Praecordial cardiograms, by offering a characteristic curve, will no doubt add to our means of diagnosing constrictive pericarditis.

\section{References}

Benchimol, A., and Dimond, E. G. (1963). The normal and abnormal apexcardiogram. Its physiologic variation and its relation to intracardiac events. American fournal of Cardiology, 12, 368.

$\longrightarrow,-$, and Carson, J. C. (196I). The value of the apexcardiogram as a reference tracing in phonocardiography. American Heart fournal, 61, 485.

Boicourt, O. W., Nagle, R. E., and Mounsey, J. P. D. (1965). The clinical significance of systolic retraction of the apical impulse. British Heart fournal, 27, 379.

Coulshed, N., and Epstein, E. J. (1963). The apexcardiogram: its normal features explained by those found in heart disease. British Heart fournal, 25, 697.

El-Sherif, A., Saad, Y., and El-Said, G. (1969). Praecordial tracings of myocardial aneurysms. British Heart fournal, 31, 357.

Evans, W. (1956). Cardiology, 2nd ed., p. 220. Butterworth, London.

Friedberg, C. K. (1966). Diseases of the Heart, 3rd ed., p. 971. Saunders, Philadelphia and London.

Friedreich, N. (1864). Zur Diagnose der Herzbeutelverwachsungen. Virchows Archiv für pathologische Anatomie und Physiologie und für klinische Medicin, 29, 296.

Gibson, R. (1959). Atypical constrictive pericarditis. In Proceedings of the British Cardiac Society. British Heart fournal, 21, 583.

Gillick, F. G. (1959). Electrokymography in pericarditis and constrictive pericarditis. In Cardiology, Vol. 3, pp. 8-56. Ed. by A. A. Luisada. McGraw, Hill, New York, Toronto, and London.

Gimlette, T. M. D. (1959). Constrictive pericarditis. British Heart fournal, 21, 9.

Ginn, W. M., Sherwin, R. W., Harrison, W. K., and Baker, B. M. (1967). Apexcardiography: use in coronary heart disease and reproducibility. American Heart fournal, 73, 168.

Hansen, A. T., Eskildsen, P., and Götzsche, H. (195I). Pressure curves from the right auricle and the right ventricle in chronic constrictive pericarditis. Circulation, 3, 881 . 
Hirschfelder, A. D. (1907). Some variations in the form of the venous pulse. A preliminary report. Bulletin of the fohns Hopkins Hospital, 18, 265.

Little, R. C. (195I). Effect of atrial systole on ventricular pressure and closure of the A-V valves. American Fournal of Physiology, 166, 289.

Luisada, A. A., and Magri, G. (1952). The low frequency tracings of the precordium and epigastrium in normal subjects and cardiac patients. American Heart fournal, 44, 545.

McKusick, V. A. (1952). Chronic constrictive pericarditis. II. Electrokymographic studies and correlations with roentgenkymography, phonocardiography, and right ventricular pressure curves. Bulletin of the fohns Hopkins Hospital, 90, 27.

Mounsey, P. (1957). Praecordial ballistocardiography. British Heart fournal, 19, 259.

- (1959). Annular constrictive pericarditis; with an account of a patient with functional pulmonary, mitral, and aortic stenosis. British Heart fournal, 21, 325.

Nixon, P. G. F., and Polis, O. (1962). The left atrial $\mathrm{X}$ descent. British Heart fournal, 24, 173.

Sawyer, C. G., Burwell, C. S., Dexter, L., Eppinger, E. C., Goodale, W. T., Gorlin, R., Harken, D. E., and Haynes, F. M. (1952). Chronic constrictive pericarditis: Further consideration of the pathologic physiology of the disease. American Heart fournal, 44, 207.
Schnabel, T. G., Jr. (1966). Constrictive (restrictive) pericarditis. Medical Clinics of North America, 50, I23I.

Sorour, A., El-Sherif, A., El-Ramly, Z., Sallam, F., Saad, Y., and El-Said, G. (1963). The diagnosis of constrictive pericarditis. Bulletin of the Egyptian Society of Cardiology, $4,3$.

Stewart, H. J., Carty, J. R., and Seal, J. R. (1943). Contributions of roentgenology to diagnosis of chronic constrictive pericarditis. American fournal of Roentgenology, Radium Therapy, and Nuclear Medicine, 49, 349.

Tafur, E., Cohen, L. S., and Levine, H. D. (1964). The normal apexcardiogram. Its temporal relationship to electrical, acoustic, and mechanical cardiac events. Circulation, 30, 381 .

White, P. D. (1951). Chronic constrictive pericarditis. Circulation, 4, 288.

Wilson, R. H., Hoseth, W., Sadoff, C., and Dempsey, M. E. (1954). Pathologic physiology and diagnostic significance of the pressure pulse tracings in the heart in patients with constrictive pericarditis and pericardial effusion. American Heart fournal, 48, 671.

Wood, F. C., Johnson, J., Schnabel, T. G., Kuo, P. T., and Zinsser, H. F. (195I). The diastolic heart beat. Transactions of the Association of American Physicians, 64, 95.

Wood, P. (I961). Chronic constrictive pericarditis. American fournal of Cardiology, 7, 48. 